extensive that it affects daily activities of the person affected, ulcers can heal and lead to disfiguring of the part of the body involved. Ghana is one of the countries in the West-Africa that buruli ulcer affects. Among the districts, our study area is affected. Unfortunately the exact way of getting the disease is not known. Our study set out to determine risk profile for transmission of $M$ Ulcerans.

Methods We conducted a case-control study with Spatial mapping, a case was defined as any person aged 2 years or more who resides in the Suhum-Kraboa-Coaltar and Akuapem South districts diagnosed of Buruli ulcer meeting the WHO clinical case definition for $M$ ulcerans disease and a control is without the disease. We carried out active case search throughout all the communities in the study area (yet to finish) and took geographical positioning system (GPS) coordinates of the cases and control as well as significant features of the environment. Culture samples of the cases will be tested to show the various haplotypes.

Results So far 50 cases and controls have been identified. GPS maps generated shows areas where buruli ulcer is most prevalent and its relation to the Densu River.

Conclusion Our preliminary findings show that there is clustering of cases of buruli ulcer. Haplotypes of the various cases are yet to be done to throw more light on the mode of transmission.

\section{SP5-33 SYSTEMATIC REVIEW OF THE ADVERSE EFFECTS OF CUTANEOUS LEISHMANIASIS TREATMENT IN THE NEW WORLD}

doi:10.1136/jech.2011.142976p.69

${ }^{1} \mathrm{~L}$ Oliveira, ${ }^{1} \mathrm{~A}$ Schubach, ${ }^{2} \mathrm{M}$ Martins, ${ }^{1} \mathrm{~S}$ Passos, ${ }^{*}{ }^{1} \mathrm{R}$ Oliveira, ${ }^{1} \mathrm{M}$ Marzochi, ${ }^{1} \mathrm{C}$ Andrade. 'Evandro Chagas Clinical Researcher Institute (IPEC)/Oswaldo Cruz Foundation (Fiocruz), Rio de Janeiro/Rio de Janeiro, Brazil; ${ }^{2}$ Public Health Library (ENSP)/Oswaldo Cruz Foundation (Fiocruz), Rio de Janeiro/Rio de Janeiro, Brazil

Introduction Pentavalent antimonials are first-line drugs for the treatment of the cutaneous form of American tegumentary leishmaniasis. Second-line drugs include amphotericin B and pentamidine. Although these drugs have been used for decades, there is still no systematic review about their safety. The objective of this review was to identify the adverse effects associated with these drugs and to estimate the frequency of these effects.

Methods Intervention studies, case series and case reports containing information regarding clinical, laboratory or electrocardiographic adverse effects of drugs used for the treatment of cutaneous leishmaniasis were systematically retrieved from 10 databases searched between 13 August 2008 and 31 March 2009.

Results The 65 studies included in this review had treated 4359 patients from 12 countries infected with 8 different Leishmania species. Despite the small number of drugs used in these studies, a wide variability in the therapeutic regimens was observed. As a consequence, the adverse effects of these drugs needed to be classified jointly according to system, irrespective of daily dose, duration of treatment and route of administration. The frequencies of adverse effects were calculated based on the data of 1866 patients. The most frequently reported clinical adverse effects of pentavalent antimonials and pentamidine were musculoskeletal pain, gastrointestinal disturbances. Electrocardiographic OTc interval prolongation and a mild increase in liver and pancreatic enzymes were additional adverse effects of pentavalent antimonials.

Conclusion Although closer surveillance is needed for the treatment of cutaneous leishmaniasis, anti-leishmanial drugs are safe and the side effects severe enough to stop treatment are uncommon.

\section{SP5-34 DETECTION OF ANTENATAL DEPRESSION AND MALE GENDER PREFERENCE IN PREGNANT WOMEN IN A TEACHING HOSPITAL-A DEVELOPING NATION SCENARIO}

doi:10.1136/jech.2011.142976p.70

V Nagaraja, ${ }^{*}$ M A Khan, G Bhat. Mysore Medical College and Research Institute, Mysore, Karnataka, India

Background Maternal depression during pregnancy has been studied less than depression in postpartum period. Prevalence rate has been found to be between $5 \%$ and $26 \%$ for antenatal depression based on very few studies done. Various psycho-social and endocrine factors have been connected to antenatal depression. Antenatal depression has also been considered to increase the risk for postnatal depression. In India, female feticide and male preference has resulted in decline of child sex ratio (933/1000 males)

Aim To identify antenatal depression and male gender preference among pregnant women.

Methods This study was done from July to September 2010. A total of 221 subjects were studied covering the sociodemographic, cultural and other risk factors including gender preference. Edinburgh postnatal depression scale was used for detection of depression.

Results $74 \%$ of the women came from rural background. Most of them were illiterate, Hindu (83\%), housewives (86\%) who lived in joint families (62\%) and had family support. Average married life was about 2 years and relationship of $75 \%$ of the women was reportedly good with their husbands. $81 \%$ of the women did not have a boy. $46 \%$ had a preference for a boy. $28 \%$ of the women were depressed.

Conclusion $28 \%$ of women were depressed and $40 \%$ of them had male gender preference.

\section{SP5-35 STIGMA AMONG THE LEPROSY PATIENTS OF URBAN LEPROSY CENTRES IN MYSORE: A FIELD STUDY}

doi:10.1136/jech.2011.142976p.71

V Nagaraja,* M A Khan, G Bhat. Mysore Medical College and Research Institute, Mysore, Karnataka, India

Aim To analyse stigma prevalence, KAP and mental state of the leprosy patients of urban population of Mysore.

Setting and Design This study is a cross sectional study done among all the leprosy patients of urban leprosy centres of Mysore.

Methods and Material All the patients who were clinically detected at U.L.C., K.R.Hospital and U.L.C., Nazarbad in Mysore were interviewed. Modified Kuppuswamy Scale was used to analyse the socio-economic status of the patients. Field work in the form of home visits were done to asses the socio economic status, living conditions and mental status of all the patients registered.

Results $52 \%$ belonged to lower socio-economic status and stigma association with disease was seen to a higher extent. Deformities were major reason for Stigma association with the disease, in majority of cases. Striking ignorance about the disease could be noted with $42 \%$ unaware of name of the disease, $30 \%$ believing the cause to be curse/wrath/sin \& a vast majority ( $80 \%$ ) ignorant of the mode of spread. A majority of the patients (54\%) showed signs of depressive mental states.

Conclusions Although much has been done by intensive therapy to decrease prevalence of leprosy, stigma still continues to persist and there's a danger of it being over sighted. Need for intensive and uninterrupted (Information, Education, Communication-IEC) activities at all levels with active government and NGO participation. Detection of disease at earliest stage \& MDT, thereby reducing deformities should be aggressively done. 